\title{
The role of awareness in anticipation and recall performance in the Hebb repetition paradigm: implications for sequence learning
}

\author{
Katherine Guérard • Jean Saint-Aubin • \\ Pierre Boucher • Sébastien Tremblay
}

Published online: 30 March 2011

(C) Psychonomic Society, Inc. 2011

\begin{abstract}
Sequence learning has notably been studied using the Hebb repetition paradigm (Hebb, 1961) and the serial reaction time (SRT) task (Nissen \& Bullemer, Cognitive Psychology 19:1-32, 1987). These two paradigms produce robust learning effects but differ with regard to the role of awareness: Awareness does not affect learning a repeated sequence in the Hebb repetition paradigm, as is evidenced by recall performance, whereas in the SRT task, awareness helps to anticipate the location of the next stimulus. In this study, we examined the role of awareness in anticipation and recall performance, using the Hebb repetition paradigm. Eye movements were monitored during a spatial reconstruction task where participants had to memorize sequences of dot locations. One sequence was repeated every four trials. Results showed that recall performance for the repeated sequence improved across repetitions for all participants but that anticipation increased only for participants aware of the repetition.
\end{abstract}

This research was supported by discovery grants from the Natural Sciences and Engineering Research Council of Canada to Jean SaintAubin and Sébastien Tremblay. While working on this article, Katherine Guérard was supported by a postdoctoral fellowship from NSERC. Thanks are due to Daniel Lafond and Ian Neath for critical reading of earlier drafts.

K. Guérard $(\bowtie) \cdot J$. Saint-Aubin $\cdot$ P. Boucher

École de psychologie, Université de Moncton,

New Brunswick E1A 3E9, Canada

e-mail: katherine.guerard@umoncton.ca

S. Tremblay

École de psychologie, Université Laval,

Québec, Canada
Keywords Sequence learning · Anticipation - Awareness . Memory $\cdot$ Hebb repetition

The capacity to learn sequences from the repeated cooccurrence of stimuli in the environment has fascinated researchers for decades. Several paradigms have been developed to understand the processes that underpin sequence learning - notably, the Hebb repetition paradigm (Hebb, 1961) and the serial reaction time (SRT) task (Nissen \& Bullemer, 1987). The Hebb repetition paradigm requires participants to memorize a series of items in their order of presentation (i.e., serial recall task). One particular sequence is repeated every three trials. Learning of the repeated sequence is demonstrated by improved recall performance (Hebb, 1961) and anticipation at encodingthat is, the landing of gaze on the area of target items prior to their occurrence (Tremblay \& Saint-Aubin, 2009). Most studies on the Hebb repetition effect suggest that the improvement in recall performance for the repeated sequence is not modulated by whether or not participants are aware of the repetition (e.g., Couture \& Tremblay, 2006; McKelvie, 1987). However, in the SRT task, awareness has been shown to improve learning: Participants aware of the repetition show more anticipatory behavior than do unaware participants (Willingham, Nissen, \& Bullemer, 1989). This discrepancy may be unfounded, since the role of awareness for anticipation in the Hebb repetition effect has never been investigated. Accordingly, we investigated the role of awareness in sequence learning, using the Hebb repetition paradigm, with measures of recall performance and anticipation.

The classic Hebb (1961) repetition effect was first demonstrated during an immediate serial recall task where participants were asked to memorize the order in which 
series of digits were presented. Immediately after the presentation of each list, participants were required to recall the digits in their presentation order. Over the course of the experiment, one list of digits - that is, the same digits in the same order-was repeated every three trials. Hebb observed that recall performance increased over the course of the experiment for the repeated sequence, but not for the non repeated sequences. Hebb measured the number of aware and unaware participants, but there was no direct comparison of sequence learning between the two groups. In this paradigm, learning of the repeated sequence is assessed through the explicit immediate recall of the repeated sequence after it has been presented. The Hebb repetition effect was first demonstrated with verbal stimuli (e.g., Hebb, 1961; McKelvie, 1987) and has recently been extended to the spatial domain (e.g., Couture \& Tremblay, 2006; Gagnon, Bédard, \& Turcotte, 2005; Tremblay \& Saint- Aubin, 2009; Turcotte, Gagnon, \& Poirier, 2005). For instance, Couture and Tremblay used the dot task developed by Jones, Farrand, Stuart, and Morris (1995), in which participants had to remember a sequence of dot locations for immediate recall. After presentation of the sequence, all the dot locations reappeared simultaneously on the computer screen, and participants had to reproduce their presentation order. Over the course of the experiment, one sequence of dot locations was repeated every four trials. As it is the case for sequences of digits (see, e.g., Hebb, 1961), Couture and Tremblay showed that performance increased for the repeated sequence, but not for the non repeated sequences. In addition, the gradient of improvement for the repeated sequence was virtually the same for digits and dot locations.

Few studies have investigated the role of awareness in the Hebb repetition effect (Couture \& Tremblay, 2006; Gagnon et al., 2005; McKelvie, 1987; Turcotte et al., 2005). Only one of these studies showed results suggesting a contribution of conscious processes in sequence learning. Gagnon's et al. aim was to test the effect of aging on sequence learning by comparing the performance of old and young adults. Of particular interest to this study was the group of young adults. Gagnon et al. either told (the intentional group) or did not tell (incidental group) participants about the presence of a repeated sequence. Participants in the intentional group were informed that a sequence would be repeated every three trials (on trials 3, 6, 9, and so on). Moreover, there was an asterisk presented before each repeated trial. Gagnon et al. showed that the repeated sequence was learned better by the intentional than by the incidental group, concluding that prior knowledge of the repeated sequence improved learning. However, 20 of the 25 participants in the incidental group reported being aware of the repetition, a ratio much higher than that reported in other studies (e.g., Couture \& Tremblay, 2006; Hebb, 1961). Therefore, the steeper rate of learning in the intentional group was due not to participants' awareness of the repetition, but to differences between the incidental and intentional learning procedures. ${ }^{1}$ This idea has been further strengthened by other studies showing no effect of awareness on the Hebb repetition effect (e.g., Couture \& Tremblay, 2006; McKelvie, 1987; Turcotte et al., 2005).

Couture and Tremblay (2006) assessed awareness at the end of the experiment by asking participants whether they had noticed anything particular during the course of the experiment, followed by a recognition test asking them to identify the repeated sequence among three distractor sequences. Participants who were categorized as aware, on the basis of their response to the initial question, showed higher recognition accuracy than did participants who were not aware of the repetition. Consistent with the results in Mckelvie (1987) and Turcotte et al. (2005), Couture and Tremblay reported that sequence learning, as assessed by immediate serial recall performance, occurred at the same rate for aware and unaware participants.

The results obtained with the Hebb repetition paradigm seem to contradict those obtained in the SRT task with regard to the role of awareness. The SRT task was developed by Nissen and Bullemer (1987). On each trial, one stimulus appeared in one of four possible locations. Participants were instructed to press the key that was located below the stimulus as quickly as possible. Over 100 trials, the stimuli could appear in random locations or as 10

\footnotetext{
${ }^{1}$ To further investigate the role of awareness in sequence learning, Gagnon et al. (2005) computed an additional analysis. At the end of the experiment, all participants in the incidental learning group performed a delayed recall of the repeated sequence. Young adults, whether they were aware or not of the repetition, were successful at the delayed recall task, with an average proportion correct of .64. The role of awareness in sequence learning was then assessed using the delayed recall score, instead of the post experimental question. Gagnon et al. reasoned that "the quite high accuracy displayed by the young adults strongly indicates that they had explicit knowledge of the sequence at the end of testing. As a result, the question that needs to be answered now is whether or not explicit knowledge of the sequence translated into elaborate encoding of the repeated sequence during the supra-span learning task" (p. 230). This possibility was investigated by correlating, for each block of trials, the delayed recall score with the repeated sequence advantage (recall of the repeated sequence minus recall of the non repeated trials in each block). Results revealed only one significant correlation for the penultimate block. The authors concluded that learning of the repeated sequence is mediated (1) by non conscious processes, because five out six correlations were not significant, and (2) by some conscious elaborate processing in the late segment of the task, because the correlation at the sixth of the seven blocks was significant. This single correlation shows that for one of the blocks, there is a link between immediate and delayed recall. This only suggests that participants who learned better the repeated series remembered it for a longer amount of time (see also Page \& Norris, 2009) and does not imply that sequence learning is different for aware and non aware participants.
} 
repetitions of a series of 10 locations. Nissen and Bullemer showed that participants' reaction times decreased drastically across blocks of repeated series but remained stable across blocks of random locations. This pattern of results has been replicated numerous times and is taken as evidence that some learning of the repeated sequence has occurred (e.g., Curran \& Keele, 1993; Elsner \& Hommel, 2001; Keller \& Koch, 2008; Willingham et al., 1989).

In several studies, researchers have investigated the role of awareness in the SRT task in order to further understand the processes involved in sequence learning. After completing the SRT task, Curran and Keele (1993) asked participants whether they thought the stimulus order followed any rule or formed any pattern. Those with positive responses were then asked to describe the pattern. Participants who were able to describe the sequence were considered "the most aware participants", while participants who did not report any pattern or those that mentioned the presence of a repeated sequence but were unable to describe the repeated pattern were classified as "the least aware participants". Curran and Keele showed stronger learning effects for more aware than for less aware participants; that is, reaction time decreased across repetitions more drastically for more aware participants. Such results have been replicated in several studies (e.g., Hoffman \& Koch, 1997; Koch, 2007; Tubau \& LópezMoliner, 2003; Willingham et al., 1989), although a few studies have shown no difference between aware and unaware participants (see Marcus, Karatekin, \& Markiewicz, 2006; Stadler, 1995).

Many researchers attribute decreased reaction time for the repeated sequence in the SRT task to learning that leads to anticipation of the location of the next stimulus (Nissen \& Bullemer, 1987; Remillard, 2003; Rüsseler, Hennighausen, \& Rösler, 2001). Remillard suggested that in a block of random stimuli, attention can be directed to the correct location only upon presentation of the stimulus, whereas in a block of repeated sequences, participants can orient their attention toward the stimulus before it is presented. According to the classic view of attention, when the stimulus is presented in the attended location, a response can be executed more quickly (Posner, 1980).

In order to account for the role of awareness in anticipation, it has been proposed that only aware participants can anticipate the location of the next stimulus. As was suggested by Willingham et al. (1989), "when subjects develop explicit [emphasis added] declarative knowledge of the sequence, they can use this knowledge to form attentional expectancies regarding the next item in the sequence" (p. 1059). Such an idea, however, is difficult to test using the SRT procedure, because anticipation is usually defined as a very rapid response that is faster than a predetermined threshold. Consequently, anticipation and responses are confounded. Short response times may reflect anticipation but may also be influenced by other factors, such as the building of stimulus-response associations. Moreover, whereas anticipation is defined as a behavior that precedes the apparition of a stimulus, responses are given after its apparition.

To circumvent the problem of defining anticipation as a fast response given after the stimulus onset, Marcus et al. (2006) introduced eye movements for investigating anticipation in the SRT task. Participants were instructed to respond by moving their eyes to the stimuli as quickly as possible upon its presentation. The author's showed that in the blocks of repeated sequences, participants moved their eyes toward the location of the stimulus before its occurrence and, of particular interest, the observed anticipatory behavior was not modulated by awareness. However, before concluding that anticipation is not modulated by awareness, three points must be addressed. First, in Marcus et al.'s study, there was an overlap between anticipation and response measures. Anticipation, which was defined as an eye movement to the location of a stimulus before it is presented, was still confounded with the response of an eye movement to the stimuli (see Tremblay \& Saint-Aubin, 2009, for a discussion). Indeed, when an eye movement was made before the presentation of the stimulus, it was considered both as a response and as anticipation. Considering the effect of awareness, it is therefore not clear whether this study assessed response time or anticipation. Second, aware participants in Marcus et al.'s study were not better at recalling the sequence after the experiment than were those who were unaware. Participants were first asked whether they had noticed something particular about the order of the stimuli. Participants who mentioned the presence of a repetition were no better at reproducing the repeated sequence or at predicting the location of the stimuli, than were participants who did not notice the repetition. Again, it is not clear whether aware participants were really different from unaware participants. Third, there were only 18 participants in Marcus et al.'s study, which may have undermined statistical power, especially in light of the fact that awareness is a between-participants factor (see Koch, 2007).

\section{The present study}

One possible reason for the discrepancy between the anticipation data in the SRT task and recall performance in the Hebb repetition paradigm (Hebb, 1961) with regard to awareness is that both measures reflect different processes. For instance, aware participants may anticipate more over repetitions because they can use an explicit memory representation to allocate their attention to the next location before its presentation (e.g., Remillard, 2003), whereas recall performance will increase at the same rate for aware and unaware participants because recall perfor- 
mance depends more on implicit learning processes. In the present study, we examined how anticipation and recall performance are modulated by awareness with the spatial Hebb repetition paradigm used by Tremblay and Saint-Aubin (2009), who showed that on the repeated trials, participants moved their eyes to the location of the dot before it was presented. Anticipation increased over repetitions, as in the case in the SRT task (e.g., Marcus et al., 2006). However, Tremblay and Saint-Aubin did not measure the participants' level of awareness; Therefore, it is unknown whether anticipation differs between aware and unaware participants.

In the present experiment, the procedure employed by Tremblay and Saint-Aubin (2009) was used. On each trial, seven black dots were presented successively at different locations on the computer screen. After the sequence, all the dots reappeared simultaneously, and participants had to recall them in the same order as that in which they had initially been presented. During the course of the experiment, the same sequence of dots was repeated every four trials. Two measures were used. First, the immediate recall performance for the repeated and non repeated trials was measured. Second, in order to measure anticipation, eye movements were recorded during the presentation of the seven dot locations, and we computed the amount of time spent on a dot location before the dot was presented.

In order to measure awareness, participants were asked if they had noticed something particular during the experiment. Awareness can be conceptualized in different ways (see Rünger \& Frensch, 2010, for a discussion). One theory is that awareness is a matter of degree and that the level of awareness is determined by the degree to which participants can have access to the target information. A second theory is that awareness is an all-or-none phenomenon and that "[conscious] content can be reported, reasoned about and used for voluntary action" (Rünger \& Frensch, 2010, p. 128). We adopted the latter definition, since it seems to be more representative of the view generally conveyed in studies on sequence learning, as evidenced by the long tradition of separating participants into two groups, those who are aware and those who are not aware. Therefore, the participants who mentioned the presence of the repeated trial were considered as aware, and the others were considered as unaware. Moreover, according to this definition, if participants are aware of the repeated sequence, they should be able to report it at the end of the experiment. A recognition test was therefore used for all participants, in which they had to choose, from four alternative sequences, which one was the repeated series. The recognition test was used to ensure that aware participants had access to the representation of the repeated sequence, in which case their recognition rate should be higher than that in the unaware group.

As was shown in previous studies using the Hebb repetition paradigm (Hebb, 1961), learning rate of the repeated sequence, as assessed by the recall performance, should be equivalent between the aware and unaware participants (e.g., Couture \& Tremblay, 2006; McKelvie, 1987). If anticipation is possible only for aware participants, anticipation should increase as a function of repetition for aware participants, but not for unaware participants.

\section{Method}

Participants Seventy-four students from the Universite de Moncton volunteered to take part in the experiment. All reported normal or corrected-to-normal vision.

Apparatus and materials Eye movements were recorded using the SR Research Ltd Eye Link II system. The system's resolution and sampling rate were $<0.5^{\circ}$, and $500 \mathrm{~Hz}$. Eye movements were captured by two cameras mounted on a headband that allowed tracking of both eyes and head position for head-motion compensation. Only the pupil of the participant's eye (for which the most accurate calibration was achieved) was tracked. Participants were seated at approximately $60 \mathrm{~cm}$ from the computer screen on which the experiment was presented. The computer screen had a resolution of $1,024 \times 768$ pixels.

The stimuli were 50 sequences of seven black dots. At a distance of $60 \mathrm{~cm}$ from the screen, the diameter of each dot sustained $1.2^{\circ}$ of visual angle. The dots were presented at different locations on the screen within an invisible window sustaining $19.5^{\circ} \times 19.5^{\circ}$ of visual angle. Thirty-seven sequences were constructed, with the restriction that each pair of dots taken two by two within a sequence was separated by at least $3.6^{\circ}$ and at most $12.3^{\circ}$. Among the 74 participants, all 37 sequences were used as a repeated sequence twice. For each participant, one sequence was repeated every four trials, with the first occurrence on trial 4 , for a total of 12 repetitions. The remaining 36 sequences served in the non repeated trials. Two filler sequences that were the same for all participants were also presented after the 48th trial, so that the experiment did not end with a repeated trial.

Procedure Participants were tested individually. The experimental session began with a calibration phase, during which participants were asked to fixate alternatively nine calibration dots. In order to ensure accuracy of eye movement recording, participants had to fixate a single calibration dot before the beginning of each trial. The calibration dots were visually distinctive from the to-beremembered (TBR) locations. On each trial, seven TBR black dots were presented successively on the computer screen at a rate of one per second ( $700 \mathrm{~ms}$ on/300 ms off). After the presentation of the last dot, all the dots reappeared 
simultaneously on the computer screen, and participants were instructed to point to the dots with their finger in the same order as that in which they had originally been presented (for similar procedures, see Gagnon et al., 2005; Tremblay \& Saint-Aubin, 2009; Turcotte et al., 2005). Their responses and eye movements were recorded while participants responded, in order to clarify any ambiguity that could have occurred during the online data scoring.

Following the memory task, participants were questioned about their awareness of the repeated trial. Participants were asked whether they had noticed anything particular during the experiment and were considered aware if they referred to the repetition of a sequence during the experiment. Whether they reported being aware of a repetition or not, they were informed that one sequence had been repeated during the course of the experiment. They were then presented with one sheet of paper showing a $2 \times 2$ matrix of four dot sequences. No order information was presented, but only seven dots marking the seven TBR locations. One pattern corresponded to the repeated sequence, while the other three patterns were non repeated sequences that had also been presented during the course of the experiment. Participants were asked to indicate which sequence had been repeated during the experiment.

\section{Results}

Awareness Forty out of 74 participants (54\%) reported being aware of the repetition, which is similar to the result reported by Hebb (1961) in his seminal paper (55\%). Thirty-six of the 40 aware participants $(90 \%)$ and 15 of the 34 unaware participants (44\%) recognized the repeated sequence accurately. A chi-square analysis confirmed that aware participants recognized the repeated sequence better than did the unaware participants, $\chi^{2}(1, N=74)=18.06, p<.001$. A binomial test, however, showed that the proportion of correct recognition in the group of unaware participants was greater than chance $(25 \%),(p<.05)$. For the remaining analyses, awareness was included as a between-subjects factor.

Recall performance Responses were scored according to a strict serial recall criterion. A response was considered correct when the dot was recalled in the same serial position as that in which it had been presented. Recall performance was first examined as a function of serial position for the last 24 trials, where the effect of repetition is well established (see, e.g., Couture \& Tremblay, 2006): Performance was pooled across the last 6 repeated trials and the last 18 non repeated trials. As is shown in Fig. 1, the serial position curves show typical primacy and recency effects. A 2 (awareness) $\times 2$ (repetition) $\times 7$ (serial position) mixeddesign analysis of variance (ANOVA) with awareness as a

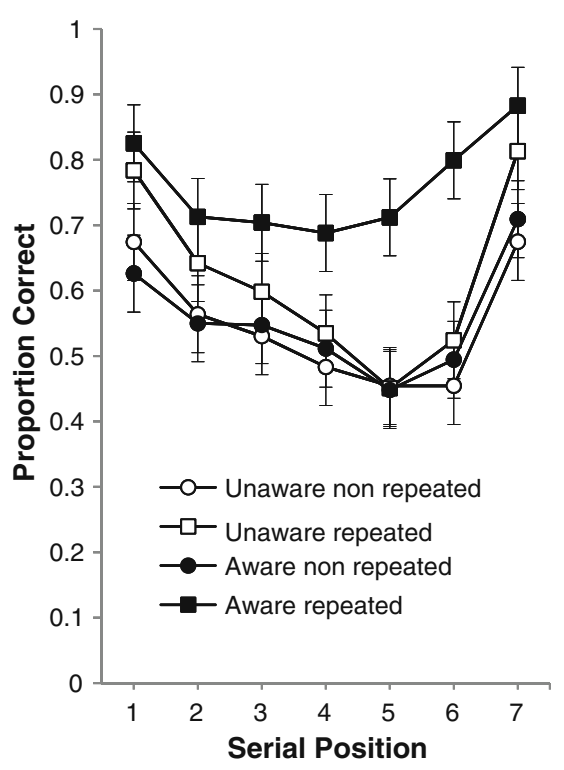

Fig. 1 Probability of correct recall as a function of serial position on the repeated and non repeated trials for aware and unaware participants. Error bars represent $95 \%$ confidence intervals based on the repeated measures factors, calculated separately for the aware and unaware groups (Masson \& Loftus, 2003)

between-subjects factor and repetition and serial position as within-subjects factors was performed on the proportion of correct recall. All analyses adopted a .05 level of significance and applied the Greenhouse-Geisser correction when the sphericity criterion was not met. The analysis showed that repeated trials were recalled better than non repeated trials, $F(1,72)=34.07, M S E=.15, \eta_{p}^{2}=.32$, that aware participants performed better than unaware participants, $F(1,72)=3.84, M S E=.36, \eta_{p}^{2}=.05$, and that recall performance varied as a function of serial position, $F(6,432)=$ 32.60, MSE $=.06, \eta_{p}^{2}=.31$. The interaction between repetition and awareness was significant, $F(1,72)=7.68$, $M S E=.15, \eta_{p}^{2}=.10$, as well as the interaction between awareness and serial position, $F(6,432)=2.93, M S E=.06$, $\eta_{p}^{2}=.04$. No other interactions were significant. Simple main effects showed that aware participants performed better than unaware participants for the repeated trials, $F(1,72)=7.21$, $M S E=.35, \eta_{p}^{2}=.09$, but not for the non repeated trials, $F<$ 1. Moreover, repeated trials were better recalled than non repeated trials for both aware, $F(1,39)=30.17, M S E=.20$, $\eta_{p}^{2}=.44$, and unaware, $F(1,33)=7.21, M S E=.09, \eta_{p}^{2}=.18$, participants.

We then computed the gradient of improvement in order to examine whether performance increased over repetitions. The 48 trials were divided into 12 blocks. For each block, we computed the recall performance for the repeated trial and the mean recall performance for the 3 preceding non repeated trials, collapsed across serial positions. For each 
participant, we computed the gradient of improvement across the 12 blocks for the repeated and non repeated trials.

In the first analysis, the 40 participants who mentioned the presence of the repeated sequence were considered aware, and the remaining 34 participants were considered unaware. As is shown in Fig. 2a, the proportion of correct responses for the repeated trials increased for both aware and unaware participants. The proportion of correct responses improved from .45 in block 1 to .68 in block 12 for the unaware participants and from .54 to .79 for the aware participants.

Averaged across participants, the proportion of correct responses in the repeated trials increased by .018 and .014 per block for aware and unaware participants, respectively. This finding is similar to that reported by Tremblay and Saint-Aubin (2009), who showed an increase of .022 per block. The proportion correct for the non repeated trials increased by .002 and .007 per block for aware and unaware participants, respectively. A $2 \times 2$ mixed-design ANOVA revealed that the gradient of improvement was
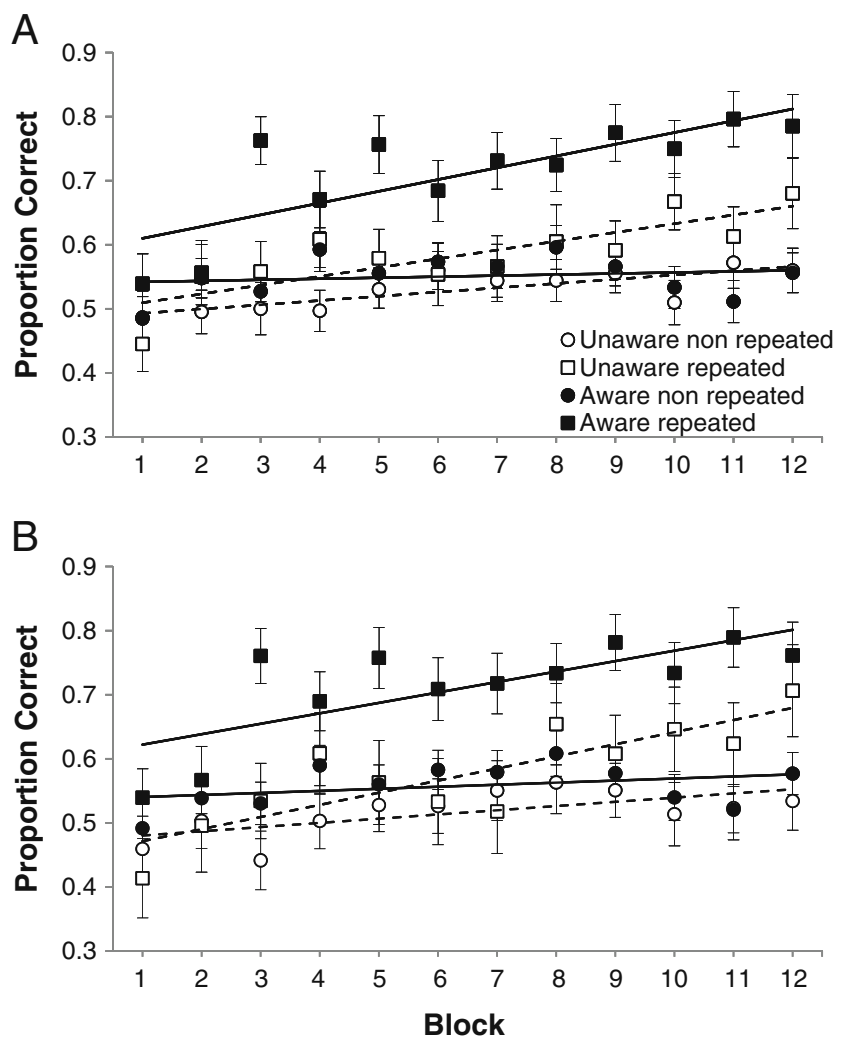

Fig. 2 Proportion of correct recall as a function of block on the repeated and non repeated trials for aware and unaware participants. a Proportion correct for the whole sample. b Proportion correct for aware participants who recognized the repeated sequence and unaware participants who did not recognize the repeated sequence accurately. Regression lines computed separately for repeated and non repeated trials, for aware (solid lines) and unaware (dashed lines) participants, are also displayed. Error bars represent the standard errors of the means higher for the repeated sequence than for the non repeated sequence, $F(1,72)=11.72, M S E=.000, \eta_{p}^{2}=.14$, but that neither the effect of awareness, $F<1$, nor the interaction between repetition and awareness, $F(1,72)=2.13, M S E=$ $.000, \eta_{p}^{2}=.03$, was significant. A linear regression was then performed on the mean proportion of correct responses in each block, averaged across all participants. The analysis, carried out separately for the repeated and non repeated trials, showed that the gradient of improvement was significant for the repeated trials, $F(1,10)=26.21, R^{2}=.72$, but not for the non repeated trials, $F(1,10)=4.59, R^{2}=.32$.

This pattern of performance indicates that both aware and unaware participants learned the repeated sequence at the same pace. One possible explanation is that our awareness criterion was not sensitive enough, implying that some participants who did not report knowledge of the repetition were in fact aware of it; that is, they may have omitted their awareness of the repetition at the end of the experiment because they were unsure about the expected answer or were afraid that they had failed to comply with the instructions. This hypothesis is supported by the fact that our unaware participants recognized the repeated pattern better than chance. As a more stringent test of awareness, the following analysis included only the 36 aware participants who recognized the repeated pattern and the 19 unaware participants who did not recognize the repeated pattern accurately.

Figure $2 b$ illustrates that the pattern of recall performance was very similar to that observed with the whole sample: The proportion of correct responses increased over blocks only for the repeated sequence, for both aware and unaware participants. The proportion of correct responses improved from .41 in block 1 to .71 in block 12 for the unaware participants and from .54 to .76 for aware participants. The proportion of correct responses for the repeated sequence increased by .016 and .019 per block for aware and unaware participants, respectively. For the non repeated trials, the proportion correct for aware and unaware participants increased by .003 and .007 , respectively, per block. A 2 (awareness) $\times 2$ (repetition) mixed ANOVA confirmed that the gradient of improvement was higher for the repeated sequence than for the non repeated sequences, $F(1,53)=9.88, M S E=.000, \eta_{p}^{2}=.16$, but that the main effect of awareness and the interaction between repetition and awareness were not significant $\left(F_{\mathrm{S}}<1\right)$. A linear regression carried out separately for the repeated and non repeated trials showed that the gradient of improvement was significant for the repeated trials, $F(1,10)=11.13, R^{2}=$ .53 , but not for the non repeated trials, $F<1, R^{2}=.00$.

Anticipation Anticipation was calculated when the participants' gaze (1) moved within the acceptance area of dot $n$ during the presentation of dot $n-1$ or during the 300 -ms blank 
interval between the offset of dot $n-1$ and the onset of dot $n$ and (2) remained within the acceptance area until dot $n$ was presented. The acceptance area was centered at the position of the dot, and its radius $\left(2.6^{\circ}\right.$ at viewing distance of $\left.60 \mathrm{~cm}\right)$ represented the middle point between the nearest two dots out of all 37 sequences, so that the acceptance areas of two dots in the same sequence never overlapped. When anticipation occurred, we calculated the amount of time the participants' gaze remained in the acceptance area of dot $n$ before dot $n$ was presented. For each of the 12 blocks, we summed the anticipation duration for all serial positions in the repeated trial and averaged the sum of anticipation for all serial positions in three non repeated trials.

In the first analysis, the 40 participants who mentioned the presence of the repeated sequence were considered as aware, and the remaining 34 participants were considered as unaware. As is illustrated in Fig. 3a, anticipation increased across blocks only for the repeated sequence for the aware participants, from $4 \mathrm{~ms}$ in block 1 to $106 \mathrm{~ms}$ in block 12. Anticipation duration in the repeated trials increased by $7.8 \mathrm{~ms}$ per block for aware participants and
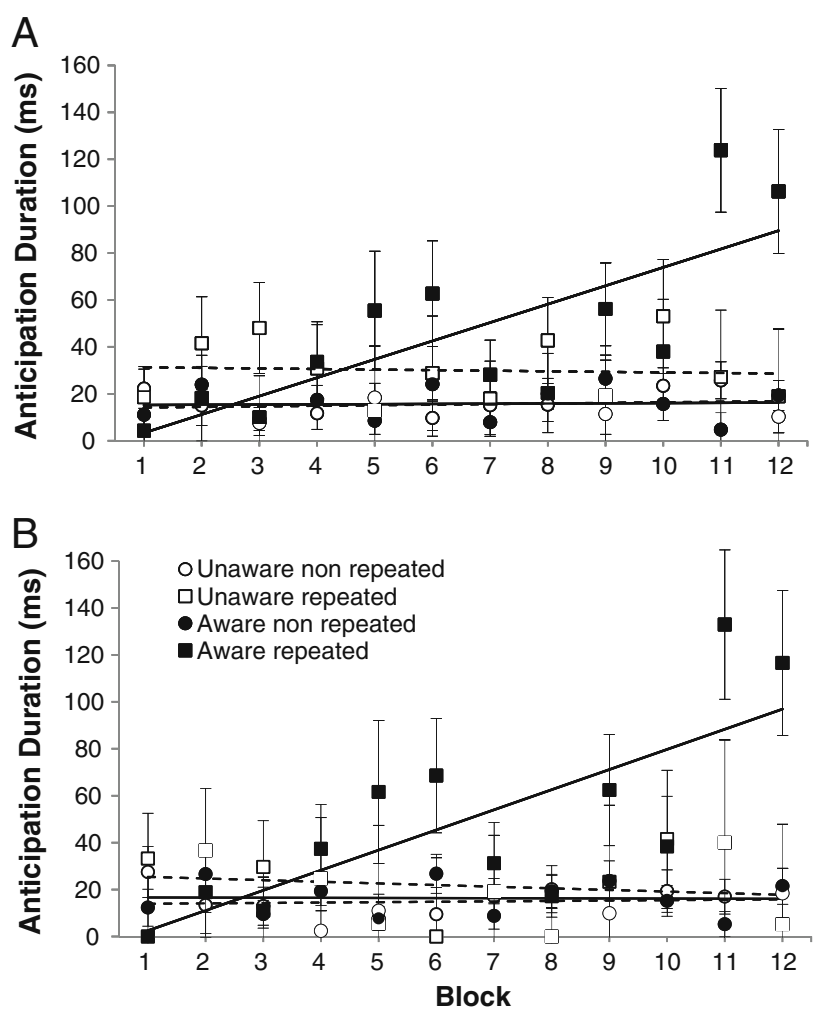

Fig. 3 Anticipation duration as a function of block in the repeated and non repeated trials for aware and unaware participants. a Anticipation for the whole sample. b Anticipation for aware participants who recognized the repeated sequence and unaware participants who did not recognize the repeated sequence accurately. Regression lines computed separately for repeated and non repeated trials, for aware (solid lines) and unaware (dashed lines) participants, are also displayed. Error bars represent the standard errors of the means decreased by $0.2 \mathrm{~ms}$ per block for unaware participants. The increase of anticipation in the repeated trials for the aware group is slightly higher than that $(6.3 \mathrm{~ms})$ observed by Tremblay and Saint-Aubin (2009). Anticipation duration in the non repeated trials diminished by 0.1 and $0.2 \mathrm{~ms}$ for aware and unaware participants, respectively. A 2 (awareness) $\times 2$ (repetition) mixed ANOVA was carried out on the gradient of improvement over blocks for anticipation duration. The analysis indicated that the gradient of improvement was higher for the repeated sequence than for the non repeated sequences, $F(1,72)=7.55, M S E=$ $64.09, \eta_{p}^{2}=.10$, and was higher for aware participants than for unaware participants, $F(1,72)=7.52, M S E=76.78$, $\eta_{p}^{2}=.10$. Most importantly, the interaction between repetition and awareness was also significant, $F(1,72)=$ 9.73, $M S E=64.09, \eta_{p}^{2}=.12$. Simple main effects showed that for aware participants, the gradient of improvement was higher for the repeated trials than for the non repeated trials, $F(1,39)=14.04, M S E=85.49, \eta_{p}^{2}=.27$, whereas it did not differ between repeated and non repeated trials for unaware participants, $F<1$. Moreover, the gradient of improvement was higher for aware participants than for unaware participants for the repeated trials, $F(1,72)=9.31$, $M S E=128.90, \eta_{p}^{2}=.12$, but not for the non repeated trials, $F<1$. A linear regression performed on the anticipation duration in each block, averaged across all participants, showed that for aware participants, the gradient of improvement was significant for the repeated trials, $F(1,10)=13.76$, $R^{2}=.58$, but not for the non repeated trials, $F<1, R^{2}=.00$. For unaware participants, the gradient of improvement was not significant for both the repeated, $F<1, R^{2}=.00$, and non repeated trials, $F<1, R^{2}=.02$.

The same analysis was repeated with the subsample composed of the 36 aware participants who had recognized the repeated pattern and of the 19 unaware participants who had not recognised the repeated pattern accurately. As is shown in Fig. 3b, anticipation duration increased as a function of block in the repeated trials only for the aware participants. Indeed, anticipation duration in the repeated trials increased by $7.8 \mathrm{~ms}$ per block for aware participants - that is, from $0 \mathrm{~ms}$ in block 1 to $117 \mathrm{~ms}$ in block 12 -and decreased by $0.7 \mathrm{~ms}$ per block for unaware participants. On the non repeated trials, anticipation duration increased by $0.2 \mathrm{~ms}$ per block for unaware participants and diminished by $0.1 \mathrm{~ms}$ per block for aware participants. A 2 (awareness) $\times 2$ (repetition) mixed ANOVA confirmed that the gradient of improvement was higher for the repeated sequence than for the non repeated sequences, $F(1,53)=4.97, M S E=75.46, \eta_{p}^{2}=.09$, and was higher for aware participants than for unaware participants, $F(1,53)=5.65, M S E=90.48, \eta_{p}^{2}=.10$. The interaction between repetition and awareness was also significant, $F(1,53)=7.44, M S E=75.46, \eta_{p}^{2}=.12$. Simple 
main effects showed that for aware participants, the gradient of improvement was larger for the repeated trials than for the non repeated trials, $F(1,35)=15.15, M S E=88.55, \eta_{p}^{2}=.30$, whereas it did not differ between repeated and non repeated trials for unaware participants, $F<1$. The gradient of improvement was larger for aware participants than for unaware participants for the repeated trials, $F(1,53)=6.93, M S E=$ $154.50, \eta_{p}^{2}=.12$, but not for the non repeated trials, $F<1$. A linear regression confirmed that for aware participants, the gradient of improvement was significant for the repeated trials, $F(1,10)=12.98, R^{2}=.57$, but not for the non repeated trials, $F<1, R^{2}=.00$. For unaware participants, the gradient of improvement was not significant for both the repeated, $F<1, R^{2}=.03$, and non repeated, $F<1, R^{2}=.01$, trials.

\section{Discussion}

The objective of this experiment was to examine the role of awareness in learning through measures of anticipation and recall performance in the Hebb repetition paradigm (Hebb, 1961) with a spatial serial recall task. We replicated the Hebb repetition effect by showing that recall performance increased over the course of the experiment for the repeated trials, but not for the non repeated trials (see, e.g., Couture \& Tremblay, 2006; Hebb, 1961; Page, Cumming, Norris, Hitch, \& McNeil, 2006; Turcotte et al., 2005). This pattern of performance was similar for both aware and unaware participants, confirming that learning, as assessed through the immediate recall of the repeated sequence, is not modulated by awareness (see, e.g., Couture \& Tremblay, 2006; McKelvie, 1987). This pattern was observed using both the traditional criterion for classifying aware and unaware participants (see, e.g., Hebb, 1961) and a stricter criterion of awareness that excluded unaware participants who recognized the repeated sequence and aware participants who did not recognize the repeated series. In addition, typical serial position curves were observed, replicating previous findings in the field (see, e.g., Jones et al., 1995). The results also revealed that the repeated series was better recalled than the non repeated series at all serial positions. Although the analysis of recall performance showed that the gradient of improvement for the repeated trials was similar for aware and unaware participants, the overall level of performance differed between the two groups. This finding, also reported by Couture and Tremblay (2006), suggests that there is a difference between aware and unaware participants. Aware participants performed better than unaware participants, even in the first block where no sequence was repeated. From our results, it is not clear whether the overall level of performance is required for producing awareness of the repetition or whether both the level of performance and awareness depend on the development of a long-term memory representation. However, the critical finding, irrespective of the baseline level of performance in Block 1 , was that performance increased at the same rate for aware and unaware participants.

As was shown by Tremblay and Saint-Aubin (2009), the analysis of eye movements revealed that participants anticipated the TBR locations during encoding, meaning that they moved their eyes to the location of the stimulus before it was presented. Although awareness did not modulate recall performance, our results indicate that anticipation for the repeated trial increased over the course of the experiment only for the participants aware of the repetition. On the one hand, the finding that anticipation duration is modulated by awareness is in line with the results of studies using the SRT task. Indeed, several studies using the SRT task showed that participants aware of the repetition had stronger learning effects than did participants unaware of the repetition (Curran \& Keele, 1993; Hoffman \& Koch, 1997; Koch, 2007; Tubau \& López-Moliner, 2003; Willingham et al., 1989).

On the other hand, our results contrast with those of Marcus et al. (2006) showing that anticipation, as assessed by eye movements, is not modulated by awareness. As was discussed in the introduction, this discrepancy might be explained by their small sample and by the fact that aware and unaware groups in their study did not differ sufficiently with regard to their level of awareness, as evidenced by their ability to correctly identify the repeated sequence after the SRT task. Therefore, we believe our results provide convincing evidence that anticipation, as measured through eye movements, is modulated by awareness.

The pattern of anticipation for aware participants in the present study is similar to that reported by Tremblay and Saint-Aubin (2009) for their whole sample. Even if Tremblay and Saint-Aubin did not assess awareness, it could be hypothesized that most of their participants were likely to have been aware of the repetition. In effect, in order to study rehearsal, they inserted a 10-s retention interval between presentation and recall in which all dot locations were visible. It is possible that this additional 10 -s period during which participants were exposed to the whole sequence might have allowed them to become aware. Our finding that anticipation is modulated by awareness, whereas recall performance is independent of awareness, is inconsistent with Tremblay and Saint-Aubin's suggestion that the two measures reflect the same processes. Whether anticipatory eye movements emerge from the same process as that underlying sequence learning or are guided by a distinct process is an issue of great theoretical importance.

The present results provide empirical support for the latter view. However, it is still possible that a single process is at the basis of recall from memory and anticipation 
behaviors. In our group of unaware participants, the level of learning was possibly not high enough to allow anticipation to occur. Indeed, one has to keep in mind that a threshold of activation along the continuum between response randomness and sequence learning - that is, anticipatory eye movements require a certain level of learning before such behavior can occur-could accommodate our findings, since the presence of thresholds is known to mimic functional dissociations (see Dunn \& Kirsner, 2003).

Another possibility is that anticipation duration, because it is a time-based measure, provides a more fine-grained assessment of learning, as compared with the proportion of correct responses. Fine-grained measures traditionally reveal effects that are otherwise not visible (see, e.g., Emmorey, Luk, Pyers, \& Bialystok, 2008; MacLeod \& Hodder, 1998; Saint-Aubin, Kenny, \& Roy-Charland, 2010). In this study, however, the recall performance shows a gradient of improvement that is not apparent from the anticipation data. Therefore, our findings do not lend support to a hypothesis based on a greater sensitivity of anticipation. Rather, our data might be taken to suggest that anticipation and recall performance reflect different processes. For instance, as will be discussed in the following paragraphs, anticipation could require processes additional to those responsible for producing improvement in recall performance.

Recall performance in the Hebb repetition paradigm (Hebb, 1961) would depend on the retrieval of the correct sequence from memory, a representation that can be strengthened by the repetition of the sequence. Such learning would be implicit and occur irrespective of whether participants are aware or not of the repetition. This idea is consistent with the theories that have been developed to account for the Hebb repetition effect. Hebb suggested that long-term learning occurs through the reinforcement of associations between item representations. Moreover, the two short-term memory models that have been extended to account for the Hebb repetition effect do not assume awareness for learning to occur (see, e.g., Burgess \& Hitch, 1999; Page \& Norris, 2009). Without recourse to the concept of awareness, it can be assumed that those models suggest similar learning processes for aware and unaware participants. In the SRT literature, implicit learning would result in the development of stimulus-stimulus associations (see Hoffman \& Koch, 1997; Koch, 2007). Therefore, both aware and unaware participants would learn stimulus-stimulus associations between items of the repeated sequence (see, e.g., Willingham \& Goedert-Eschmann, 1999).

Anticipation, however, would recruit additional processes that are dependent on awareness. One possibility is that anticipation reflects attentional processes that help prepare the participants to respond to the stimuli that will be presented (see, e.g., Remillard, 2003; Willingham et al., 1989). This idea is similar to endogenous cuing, where participants voluntarily move their attention toward a stimulus (see Posner, 1980), which is thought to depend on conscious processes (see, e.g., Sumner \& Husain, 2008). Therefore, anticipation may be based on the conscious and voluntary orienting of attention. In the SRT task, awareness would allow participants to use their representation of the repeated trial to anticipate and move their attention toward the location of the to-be-presented stimulus (see, e.g., Remillard, 2003). In the Hebb repetition effect (Hebb, 1961), such voluntary shifts of attention would not be related to recall performance, since it is not modulated by awareness. Participants aware of the repetition might nevertheless use their memory representation to anticipate the location of the next stimulus as a verification strategy - a correct anticipation indicating that the repeated sequence has been correctly encoded. Therefore, anticipation would result from the conscious and voluntary use of the memory representation built from the repetition of the sequence.

Another possibility suggested in the SRT literature (Koch, 2007) is that in addition to stimulus-stimulus associations, aware participants learn response-response associations (see also Willingham \& Goedert-Eschmann, 1999; Zirngibl \& Koch, 2002). These response-response associations would take the form of a motor program corresponding to the repeated sequence that runs independently of the perceived input (see Hoffman \& Koch, 1997; Koch, 2007; Zirngibl \& Koch, 2002). This motor program would not be specific to one effector and could be used to guide manual responses, as well as eye movements (see, e.g., Willingham, 1998). According to this theory, anticipation would be a consequence of triggering a motor program corresponding to the to-be-presented sequence at the time of encoding (in the Hebb repetition paradigm) or at the beginning of the repeated series (in the SRT task).

One concern with respect to the present findings is whether they generalize to sequence learning in generalthat is, when the material does not involve visuospatial stimuli. Many lines of evidence suggest that the same memory processes are involved in verbal and visual/spatial short-term ordered recall tasks (Jones et al., 1995; Ward, Avons, \& Melling, 2005). In the case of serial learning in particular, the visuospatial version of the Hebb repetition effect has been found to be functionally equivalent to the verbal version (Couture \& Tremblay, 2006). In addition, although anticipation may take different forms depending on the type of stimuli being processed, it seems to apply to verbal material. Indeed, Eimer, Goschke, Schlaghecken, and Stürmer (1996) measured ERPs during the SRT paradigm, using verbal stimuli. They showed that the brain activation associated with the to-be-presented letter could occur within $100 \mathrm{~ms}$ after stimulus onset and even prior to the stimulus presentation. This pattern, which was inter- 
preted as evidence of anticipation, is observed only for participants who become aware of the repetition. Therefore, it is safe to conclude that explicit knowledge of the sequence can facilitate the anticipation of to-be-presented stimuli irrespective of their identity.

In conclusion, in the Hebb repetition paradigm (Hebb, 1961), learning can occur irrespective of participants' awareness. Such learning could take the form of stimulusstimulus associations, consistent with both theories of learning in the SRT literature (see, e.g., Koch, 2007) and the Hebb repetition paradigm (see, e.g., Hebb, 1961). In addition to this implicit learning, awareness would involve additional processes that allow one to voluntary move attention to the location of the next to-be-presented item (see, e.g., Remillard, 2003) or that involve the formation of response-response associations (see, e.g., Koch, 2007). Could these processes be involved in sequence learning in both SRT and the Hebb repetition paradigm? Our results indicate that anticipation in the Hebb repetition paradigm is consistent with the pattern of reaction times (e.g., Curran \& Keele, 1993) and mimics the pattern of eye movements observed in the SRT task (e.g., Marcus et al., 2006). Moreover, the modulation of anticipation by awareness previously observed in the SRT task generalizes to the Hebb repetition paradigm. These functional similarities can be taken as a suggestion that common processes are involved in sequence learning evidenced by the SRT and the Hebb repetition paradigms.

\section{References}

Burgess, N., \& Hitch, G. (1999). Memory for serial order: A network model of the phonological loop and its timing. Psychological Review, 106, 551-581. doi:10.1037//0033-295X.106.3.551.

Couture, M., \& Tremblay, S. (2006). A Hebb repetition effect for visuo-spatial information. Memory \& Cognition, 34, 1720-1729.

Curran, T., \& Keele, S. W. (1993). Attentional and nonattentional forms of sequence learning. Journal of Experimental Psychology: Learning, Memory, and Cognition, 19, 189-202. doi:110.1037/ 0278-7393.1019.1031.1189.

Dunn, J. C., \& Kirsner, K. (2003). What can we infer from double dissociations? Cortex, 39, 1-7.

Eimer, M., Goschke, T., Schlaghecken, F., \& Stürmer, B. (1996). Explicit and implicit learning of event sequences: Evidence from eventrelated brain potentials. Journal of Experimental Psychology: Learning, Memory, and Cognition, 22, 970-987. doi:10.1037// 0278-7393.22.4.970.

Elsner, B., \& Hommel, B. (2001). Effect anticipation and action control. Journal of Experimental Psychology. Human Perception and Performance, 27, 229-240. doi:210.1037/AM10961523.1027.1031.1229.

Emmorey, K., Luk, G., Pyers, J. E., \& Bialystok, E. (2008). The source of enhanced cognitive control in bilinguals. Psychological Science, 19, 1201-1206. doi:10.1111/j.1467-9280.2008.02224.x.

Gagnon, S., Bédard, M. J., \& Turcotte, J. (2005). The effect of old age on learning of supra-span spatial sequences under intentional and incidental encoding instructions. Brain and Cognition, 59, 225235. doi:10.1016/j.bandc.2005.07.001.
Hebb, D. O. (1961). Distinctive features of learning in the higher animal. In J. F. Delafresnaye (Ed.), Brain mechanisms and learning: A symposium (pp. 37-51). Oxford: Blackwell.

Hoffmann, J., \& Koch, I. (1997). Stimulus-response compatibility and sequential learning in the serial reaction time task. Psychological Research, 60, 87-97. doi:10.1007/BF00419682.

Jones, D., Farrand, P., Stuart, G., \& Morris, N. (1995). Functional equivalence of verbal and spatial information in serial short-term memory. Journal of Experimental Psychology: Learning, Memory, and Cognition, 21, 1008-1018. doi:10.1037/0278-7393.21.4.1008.

Keller, P. E., \& Koch, I. (2008). Action planning in sequential skills: Relations to music performance. The Quarterly Journal of Experimental Psychology, 61, 275-291. doi:210.1080/ 17470210601160864.

Koch, I. (2007). Anticipatory response control in motor sequence learning: Evidence from stimulus-response compatibility. Human Movement Science, 26, 257-274. doi:210.1016/j.humov.2007.1001.1004.

MacLeod, C. M., \& Hodder, S. L. (1998). Presenting two incongruent color words on a single trial does not alter Stroop interference. Memory \&Cognition, 26, 212-219.

Marcus, D. J., Karatekin, C., \& Markiewicz, S. (2006). Oculomotor evidence of sequence learning on the serial reaction time task. Memory \& Cognition, 34, 420-432.

Masson, M. E. J., \& Loftus, G. R. (2003). Using confidence intervals for graphically based data interpretation. Canadian Journal of Experimental Psychology, 57, 203-220.

McKelvie, S. J. (1987). Learning and awareness in the Hebb digits task. The Journal of General Psychology, 114, 75-88.

Nissen, M. J., \& Bullemer, P. (1987). Attentional requirements of learning: Evidence from performance measures. Cognitive Psychology, 19, 1-32. doi:10.1016/0010-0285(1087)90002-90008.

Page, M. P. A., \& Norris, D. (2009). Is there a common mechanism underlying word-form learning and the Hebb repetition effect? Experimental data and a modelling framework. In A. Thorn \& M. P. A. Page (Eds.), Interactions between short-term and long-term memory in the verbal domain (pp. 136-156). Hove, U.K: Psychology Press.

Page, M. P. A., Cumming, N., Norris, D., Hitch, G., \& McNeil, A. (2006). Repetition learning in the immediate serial recall of visual and auditory materials. Journal of Experimental Psychology: Learning, Memory, and Cognition, 32, 716-733. doi:10.1037/02787393.32.4.716.

Posner, M. I. (1980). Orienting of attention. The Quarterly Journal of Experimental Psychology, 32, 3-25.

Remillard, G. (2003). Pure perceptual-based sequence learning: A role for visuospatial attention. Journal of Experimental Psychology: Learning, Memory, and Cognition, 35, 528-541. doi:510.1037/ a0014646.

Rünger, D., \& Frensch, P. A. (2010). Defining consciousness in the context of incidental sequence learning: Theoretical considerations and empirical implications. Psychological Research, 74, 121-137. doi:10.1007/s00426-008-0225-8.

Rüsseler, J., Hennighausen, E., \& Rösler, F. (2001). Response anticipation processes in the learning of a sensorimotor sequence: Evidence from the lateralized readiness potential. Journal of Psychophysiology, 15, 95-105.

Saint-Aubin, J., Kenny, S., \& Roy-Charland, A. (2010). The role of eye movements in the missing-letter effect revisited with the rapid serial visual presentation procedure. Canadian Journal of Experimental Psychology, 64, 47-52. doi:10.1037/a0016850.

Stadler, M. A. (1995). Role of attention in implicit learning. Journal of Experimental Psychology: Learning, Memory, and Cognition, 21, 674-685. doi:10.1037//0278-7393.21.3.674.

Sumner, P., \& Husain, M. (2008). At the edge of consciousness: Automatic motor activation and voluntary control. The Neuroscientist, 14, 474-486. doi:10.1177/1073858408314435. 
Tremblay, S., \& Saint-Aubin, J. (2009). Evidence of anticipatory eye movements in the spatial Hebb repetition effect: Insights for modeling sequence learning. Journal of Experimental Psychology: Learning, Memory, and Cognition, 35, 1256-1265. doi:1210.1037/a0016566.

Tubau, E., \& López-Moliner, J. (2003). Spatial interference and response control in sequence learning: the role of explicit knowledge. Psychological Research, 68, 55-63. doi:10.1007/ s00426-00003-00139-00424.

Turcotte, J., Gagnon, S., \& Poirier, M. (2005). The effect of old age on learning of supra-span sequences. Psychology and Aging, 20, 251-260. doi:10.1037/0882-7974.20.2.251.

Ward, G., Avons, S. E., \& Melling, L. (2005). Serial position curves in short-term memory: Functional equivalence across modalities. Memory, 13, 308-317. doi:10.1080/09658210344000279.
Willingham, D. B. (1998). A neuropsychological theory of motor skill learning. Psychological Review, 105, 558-584. doi:510.1037/ 0033-1295X.1105.1033.1558.

Willingham, D. B., \& Goedert-Eschmann, K. (1999). The relation between implicit and explicit learning: Evidence for parallel development. Psychological Science, 10, 531-534. doi:510.1111/ 1467-9280.00201.

Willingham, D. B., Nissen, M. J., \& Bullemer, P. (1989). On the development of procedural knowledge. Journal of Experimental Psychology: Learning, Memory, and Cognition, 15, 1047-1060. doi:1010.1037/0278-7393.1015.1046.1047.

Zirngibl, C., \& Koch, I. (2002). The impact of response mode on implicit and explicit sequence learning. Experimental Psychology, 49, 153162. doi:110.1027//1618-3169.1049.1022.1153. 